\title{
2018 - das Jahr, in dem die deutsche Polizei erstmals Drohnen gegen Gefährder einsetzte
}

\author{
Anmerkungen zu Art. 47 Bayerisches Gefahrenabwehrgesetz (PAG)
}

Susanne Benöhr-Laqueur, Abteilung Polizei, Fachhochschule für öffentliche Verwaltung NRW/Standort Münster, Nevinghoff 8-10, 48147 Münster

(susanne.benoehr-laqueur@fhoev.nrw.de), (1) orcid.org/0000-0002-5490-113

Das neue bayerische Polizeigesetz betritt im Hinblick auf den Einsatz von Polizeidrohnen juristisches Neuland. Hochkomplexe juristische Tatbestände ermöglichen es der bayerischen Polizei in Zukunft, nicht nur öffentliche Veranstaltungen oder Ansammlungen auf großen bzw. unübersichtlichen Örtlichkeiten zu überwachen, sondern auch ganz gezielt potenzielle Gefährder zu identifizieren. Hinzu kommt, dass der Drohneneinsatz über polizeirelevanten „Hotspots“ wie Rotlichtbezirken, Hauptbahnhöfen aber auch Asylbewerbertreffpunkten unter dem Aspekt der Abwehr des neu konzipierten Tatbestandes der „drohenden Gefahr" ermöglicht wird.

\section{8 - the year in which German police first used drones against potential perpetrators \\ Comments on Art. 47, Bavarian Police Law (PAG)}

The new Bavarian police law breaks new legal ground regarding the use of police drones. Highly complex legal offenses will allow the Bavarian police in the future not only to monitor public events or gatherings at large or complex sites but also to specifically identify potential perpetrators. In addition, the use of drones over relevant "hot spots" such as red-light districts, railway stations, or meeting places for asylum seekers will be possible in defense against the newly outlined offense of "impending danger".

Keywords: unmanned aerial vehicle (UAV), police drone, chilling effect, Bavarian Police Law (PAG)

\section{Einführung}

Die deutsche Polizei nutzt seit circa einem Jahrzehnt Drohnen. Aufgrund der unausgereiften Technik war die Akzeptanz, ein Unmanned Aerial Vehicle (UAV) einzusetzen, zunächst gering. Zum gegenwärtigen Zeitpunkt haben lediglich die Bundesländer Thüringen, Hamburg und Bremen noch keine Polizeidrohne erworben. Im Bedarfsfall werden sie offenbar kurzerhand von den anderen Bundesländern ausgeliehen (Bremische Bürgerschaft 2014, S. 4565-4566). Dabei sind die Vorteile des Polizeidrohneneinsatzes in bestimmten Bereichen evident. Drohnen können Tatorte millimetergenau vermessen, Fluchtwege von Tätern nachvollziehen, den Verkehr überwachen und lenken sowie vermisste Personen suchen (Schulz 2017, S. 17). Angesichts der Tatsache, dass sie extrem leise sind, ihr Radius je nach Modell bis zu $2 \mathrm{~km}$ beträgt und sie immerhin - ohne dass die Leistungsfähigkeit der Hightechkamera beeinträchtigt wird - bis zu 1.000 Meter aufsteigen können, ist ihre Effektivität im Bereich der Beweissicherung und Überwachung unbestritten. Lediglich der Anschaffungspreis von bis zu 40.000 Euro und die kurze Flugdauer von circa 20 Minuten pro Akkuladung trüben das Bild (Diehl 2010). Dies sind jedoch Marginalien, denn angesichts der Terroranschläge in den vergangenen Jahren, der Eskalationen während des G20-Gipfels 2017 in Hamburg oder gewaltbereiter Fußballfans genießt die Sicherheitspolitik absolute Priorität. So war die Verschärfung des bayerischen Polizeigesetzes sowie die Ad-hoc-Anschaffung mehrerer Polizeidrohnen zur Jahreswende 2017/2018 keine wirkliche politische Überraschung (Truscheit 2017). Bemerkenswert ist hingegen die Signalwirkung, die vom neuen Bayerischen Polizeiaufgabengesetz im Allgemeinen und bezüglich des Einsatzes von Drohnen im Besonderen ausgeht (Kornmeier 2018).

Die Verwendung von Polizeidrohnen ist durchaus keine Novität. Bereits im Jahre 2010 wurde im Zuge der Demonstrationen gegen den Castor-Transport in Gorleben eine Aufklärungsdrohne von der Polizei eingesetzt (Hertwig und Kuvvet 2011).
This is an article distributed under the terms of the Creative Commons Attribution License CCBY 4.0 (https://creativecommons.org/licenses/by/4.0/)

https://doi.org/10.14512/tatup.27.3.14

Submitted: 20.07.2018. Peer reviewed. Accepted: 11.10.2018 
Die - nicht genehmigte - polizeiliche Nutzung hatte weitreichende politische Konsequenzen: Hinfort verzichteten die Polizeiführungen der Länder ausdrücklich auf den Drohneneinsatz über Menschenansammlungen. Besonders eindrücklich äußerte sich im Jahre 2016 der Polizeipräsident von Berlin: ,[...] unsere Drohnen fliegen nicht über Menschen und deswegen werden sie niemals für die Überwachung eingesetzt werden" (Abgeordnetenhaus Berlin 2016, S. 37). Diese Ansicht wird von der bayerischen Landesregierung nicht geteilt. Der neugeschaffene Art. 47 im Bayerischen Polizeiaufgabengesetz ${ }^{1}$ (PAG) mit der Überschrift „Einsatz von unbemannten Luftfahrtsystemen“ (Abb. 1) gestattet es der Polizei, Drohnen in einer Fülle von Fällen, wie etwa bei Demonstrationen oder Fußballspielen, aufsteigen zu lassen. Hinzu kommen Einsätze zur Identifizierung von Personen, von denen eine Gefahr für die öffentliche Sicherheit ausgehen könnte - sogenannte Gefährder. Aber auch Straftäter können zukünftig durch Polizeidrohnen observiert und belauscht werden. Schlussendlich können Polizeidrohnen auch über polizeilichen Hotspots erscheinen. Dies sind Orte, an denen sich z. B. potenzielle Gefährder aufhalten, wie etwa Rotlichtbezirke, Ausländerämter, Asylbewerberheime oder Hauptbahnhöfe.

Angesichts der Komplexität des Art. 47 PAG beschränkt sich die folgende Untersuchung auf zwei Themenbereiche. Zum einen soll untersucht werden, ob und wann die bayerische Polizei Gefährder, Demonstrationen, Fußballspiele und Asylbewerberheime mit Drohnen überwachen kann. Zum zweiten wird analysiert, über welche technische Ausrüstung eine Drohne verfügen darf. Diese Betrachtungen werden ergänzt um die Fragestellung, ob die Möglichkeiten des Drohneneinsatzes nicht den ersten Schritt in den „Orwellschen Überwachungsstaat“ darstellen.

\section{Außerordentlich komplex}

\section{Art. 47 PAG}

Bereits in der parlamentarischen Anhörung zum PAG erklärte ein Abgeordneter, dass er den Gesetzentwurf als ,,außerordentlich komplex“ empfände (Bayerischer Landtag 2018a, S. 23). Dieser Einschätzung ist vorbehaltlos zuzustimmen und insbesondere Art. 47 PAG ist hierfür ein prägnantes Beispiel. Der bayerische Gesetzgeber stand nämlich erstens vor der Aufgabe, das Polizeiaufgabengesetz einer EU-Richtlinie anzupassen, zweitens die Vorgaben des Bundesverfassungsgerichts umzusetzen und drittens dem Stand der Technik entsprechende Ergänzungen vorzunehmen (Bayerischer Landtag 2018 b, Anlage 2).

Im Hinblick auf den Einsatz von Polizeidrohnen wagt Bayern in mehrfacher Hinsicht ab sofort einen Alleingang. In den Polizeigesetzen derjenigen Länder, die Drohnen einsetzen, finden sich keine spezialgesetzlichen Normen, die den Einsatz legitimieren (Weiner 2018). Die Polizeiführungen sind somit gezwun-

1 Gesetz über die Aufgaben und Befugnisse der Bayerischen Staatlichen Polizei (Polizeiaufgabengesetz - PAG), in der Fassung der Bekanntmachung vom 14. September 1990 (GVBI. S. 397), BayRS 2012-1-1-I.

\section{Art. 47 Einsatz von unbemannten Luftfahrtsystemen}

(1) Bei den nachfolgenden Maßnahmen dürfen Daten unter den dort genannten Voraussetzungen auch durch den Einsatz unbemannter Luftfahrtsysteme erhoben werden:

1. offene Bild- und Tonaufnahmen oder -aufzeichnungen nach Art. 33 Abs. 1 bis 3,

2. Einsatz besonderer Mittel der Datenerhebung nach Art. 36 Abs. 1 ,

3. Einsatz technischer Mittel in Wohnungen nach Art. 41 Abs.1,

4. Eingriffe in den Telekommunikationsbereich nach Art. 42 Abs. 1 bis 5 und

5. verdeckter Zugriff auf informationstechnische Systeme nach Art. 45 Abs. 1 und 2.

(2) 1 In den Fällen des Abs. 1 Nr. 1 dürfen unbemannte Luftfahrtsysteme nur dann eingesetzt werden, wenn die Offenheit der Maßnahme gewahrt bleibt. $\mathbf{2}$ In diesen Fällen soll auf die Verwendung unbemannter Luftfahrtsysteme durch die Polizei gesondert hingewiesen werden.

(3) Soweit in den Fällen des Abs. 1 eine richterliche Anordnung erforderlich ist, muss diese auch den Einsatz von unbemannten Luftfahrtsystemen umfassen.

(4) Diese unbemannten Luftfahrtsysteme dürfen nicht bewaffnet werden.

Abb.1: Art. 47 Bayerisches Polizeiaufgabengesetz.

Quelle: http://www.gesetze-bayern.de/Content/Document/BayPAG-47

gen, auf allgemeine Ermächtigungsgrundlagen zurückzugreifen. In der juristischen Fachwelt ist dies umstritten. Einerseits wird die Meinung vertreten, dass zwar ein Drohneneinsatz nicht generell unzulässig sei, er jedoch angesichts des Mangels einer Ermächtigungsgrundlage nicht zum Standardinstrument im Polizeidienst avancieren sollte (Gusy 2014). Anderer Ansicht zufolge ist die Drohne durchaus ein sinnvolles Instrumentarium und der Einsatz durch die jeweilige Ermächtigungsgrundlage gedeckt (Zöller und Ihwas 2014). Gerichtsurteile zum Polizeidrohneneinsatz sind in der Bundesrepublik Deutschland - soweit ersichtlich - (noch) nicht existent. Zur rechtlichen Bewertung eines Drohneneinsatzes muss folglich ein Rückgriff auf Gerichtsentscheidungen erfolgen, in denen über den Einsatz mobiler polizeilicher Überwachungseinheiten mit herkömmlichen Technologien geurteilt wurde.

Für diese Sachverhalte hat die Rechtsprechung klare Regelungen entwickelt. Demzufolge sind z. B. verdeckte Einsätze - und damit Aufnahmen von Personen auf Demonstrationen verboten. Erst wenn Straftaten unmittelbar bevorstehen, können die Kameras ausgefahren und kann gefilmt werden. ${ }^{2}$ Fraglich ist jedoch, ob dieser Rückgriff auf die herrschende Rechtsprechung im Hinblick auf Polizeidrohnen überhaupt sinnvoll ist. Denn mobile Überwachungsfahrzeuge und mehr noch Polizisten mit High-

2 OVG Rheinland-Pfalz, in: NVWZ-RR 2015, S.570 ff. und OVG Lüneburg, in: NVWZ-RR 2015, S. $98 \mathrm{ff}$. 
techkameras am Stativ bzw. Helm sind den Regeln der Schwerkraft unterworfen. Für sie gilt, dass der Einsatz entweder nur „offen“ oder ,verdeckt" erfolgen kann - für Drohnen hingegen gelten andere physikalische Voraussetzungen.

Das war dem bayerischen Gesetzgeber durchaus bewusst, daher wählte er eine gewandte Gesetzeskombination, mit der er den „offen-verdeckten“ Einsatz von Polizeidrohnen gesetzlich zu legitimieren versucht.

\section{Von Gefährdern sowie unübersichtlichen und großen Örtlichkeiten}

\section{Art. 47 Abs. 1 Nr. 1 i. V.m. Art. 33 Abs. 1 Nr. 1 und Nr. 2 PAG}

In der Bundesrepublik Deutschland sind die Versammlungsfreiheit (Art. 8 GG) und die Meinungsfreiheit (Art. 5 GG) ein bedeutsames demokratisches Grundprinzip. So entfaltet der „Brokdorf-Beschluss“ des Bundesverfassungsgerichts aus dem Jahre 1985 bis zum heutigen Tage seine Geltung. ${ }^{3}$ Er besagt, dass es zu den unentbehrlichen Funktionselementen eines demokratischen Gemeinwesens gehört, dass der Bürger durch Ausübung der Versammlungsfreiheit aktiv am politischen Meinungsbildungs- und Willensbildungsprozess teilnehmen kann. Der Schutz der Versammlungsfreiheit sei auch dann zu erhalten, wenn mit Ausschreitungen durch Einzelne oder Minderheiten zu rechnen ist. Angesichts dessen müssen sich einschränkende behördliche Maßnahmen am Grundsatz der Verhältnismäßigkeit messen lassen. ${ }^{4}$ Dies gilt insbesondere auch für die bild- und tongebende Überwachung von öffentlichen Versammlungen - also etwa Demonstrationen oder Kundgebungen. Vor diesem Hintergrund dürfen Video- und Tonaufzeichnungen erst dann durchgeführt werden, wenn von der Polizei gewalttätige Ausschreitungen beobachtet werden. ${ }^{5}$

Damit soll der sogenannte Chilling Effect vermieden werden. Unter diesem Begriff versteht man die Selbstbeschränkung des Bürgers an einer öffentlichen Versammlung nicht teilzunehmen, weil die Befürchtung überhandnimmt gefilmt zu werden und infolgedessen soziale und berufliche Repressalien zu erleiden. Dies hätte im Extremfall zur Folge, dass sich Bürger nicht mehr aktiv am politischen Willensbildungs- und Meinungsbildungsprozess beteiligen (Assion 2014). Diese Konfliktsituation in der die Bürger aus Angst vor dem allmächtigen „Orwellschen Überwachungsstaat" von ihrem Grundrecht nicht mehr Gebrauch machen - ist nach der Rechtsprechung des Bundesverfassungsgerichts unbedingt zu vermeiden. ${ }^{6}$ Folglich haben zahlreiche Gerichte das polizeiliche Filmen von (friedlichen) Demonstranten nur unter sehr engen Voraussetzungen und auch nur als „Übersichtsaufnahmen“" gestattet.

3 BVerfGE 69, 315 (Brokdorf)

4 ebd.

5 OVG Rheinland-Pfalz, in: NVwZ-RR 2015, S. 570 ff. und OVG Lüneburg,

in: NvWZ-RR 2015, S. $98 \mathrm{ff}$.

6 BVerfGE 69, 315 (Brokdorf).
Das neue bayerische Polizeiaufgabengesetz umgeht diese verfassungsrechtlichen Vorgaben, indem es zwei Normen geschickt kombiniert. Gemäß Art. 33 Absatz 1 Nr. 1 PAG (Abb. 2), der auf den Art. 47 Abs. 1 Nr. 1 PAG verweist, können von Personen, die von der Polizei als Gefährder eingestuft werden und sich auf einer bzw. im Zusammenhang mit einer öffentlichen Veranstaltung oder Ansammlung, d. h. in deren Umfeld bzw. auf der Anreise, aufhalten oder befinden, und von denen zu befürchten ist, dass sie erhebliche Ordnungswidrigkeiten bzw. Straftaten begehen, sowohl Bild- und Tonaufnahmen als auch (Video-)Aufzeichnungen gefertigt werden, um deren Identität festzustellen. Das bedeutet, dass eine Drohnenüberwachung von Demonstrationen als auch von Veranstaltungen - wie etwa Fußballspielen mit Gefährderpotenzial problemlos möglich ist.

Darüber hinaus erfasst Art. 33 Absatz 1 Nr. 2 PAG aber auch öffentliche Veranstaltungen und Ansammlungen, die wegen der Größe oder Unübersichtlichkeit der Örtlichkeit überwacht werden müssen. Die Entscheidung, ob eine Örtlichkeit diesen Voraussetzungen entspricht, trifft die Polizeiführung. Dies bedeutet de facto, dass sich eigentlich jeder Einsatz von Polizeidrohnen im Zuge von Art. 33 Abs. 1 PAG begründen lässt. Mehr noch: Bei einer unübersichtlichen Örtlichkeit - etwa einem Innenstadtbereich - oder einer großen Örtlichkeit - z. B. einer Festwiese

\section{Das neue bayerische Polizei-}

\section{aufgabengesetz umgeht verfassungs- rechtliche Vorgaben hinsichtlich}

\section{Versammlungsfreiheit sowie politi-} scher Meinungs- und Willensbildung.

oder dem Innenraum bzw. Umfeld eines Stadions - kann der Einsatz zunächst durch Art. 33 Abs. 1 Nr. 2 PAG legitimiert werden; sollten sich sodann Gefährder zeigen, kann problemlos auf Art. 33 Abs. 1 Nr. 1 PAG zurückgegriffen werden. Es ist somit absehbar, dass der Drohneneinsatz zur polizeilichen Standardmaßnahme avancieren wird.

\section{Der Drohneneinsatz: Art. 47 Abs. 2 PAG}

Gleichwohl ist auch der bayerische Gesetzgeber an die Rechtsprechung gebunden, die im Hinblick auf die Überwachungsmaßnahmen urteilt, dass diese nur „offen“ erfolgen dürfen. Fraglich ist somit, wann ein Drohneneinsatz als „offen“ zu qualifizieren ist. Art. 47 Absatz 2 PAG normiert, dass Drohnen nur offen aufsteigen dürfen. „Offen“ bedeutet gemäß des Gesetzentwurfes, dass entweder im Eingangsbereich der Veranstaltung auf den Drohneneinsatz hingewiesen wird bzw. der Polizist, der die Drohne steuert, mit einem gut sichtbaren Hinweis auf der Kleidung versehen ist (Bayerischer Landtag 2018c, S. 68). 
Diese Regelung ist ausgesprochen interpretationsbedürftig. Denn Art. 33 Abs. 1 Nr. 1 und Nr. 2 PAG erlauben den Einsatz von Drohnen auch ,im Zusammenhang“ mit einer öffentlichen Veranstaltung oder Ansammlung. Das bedeutet, dass die Drohne nicht unbedingt direkt am Versammlungs- oder Veranstaltungsort „offen“ eingesetzt werden muss. Wenn dies der Fall ist, dann sind die folgenden Effekte zu bedenken. Sollte ein „Drohnenpolizist" gesichtet werden, wird diese Nachricht in Sekundenschnelle verbreitet. In dieser Situation werden Gefährder eine Identifizierung aus der Luft zu vermeiden wissen, indem sie unverzüglich zur Vermummung greifen. Zum zweiten könnte der Drohneneinsatz bei friedlichen Demonstranten den Chilling Effect und Ängste vor Gewalttätigkeiten auslösen. Der Demonstrant hat nämlich keine Kenntnis darüber, warum die Polizei die Drohne aufsteigen lässt. In Betracht käme ein konfliktreiches Worst-Case-Szenario aber gegebenenfalls auch nur eine einfache Überwachungsmaßnahme aufgrund der Örtlichkeit.

Dieser Argumentation ist entgegenzuhalten, dass die Polizei sich bei weitem nicht nur der Drohnenüberwachung bedient. Überwachungswagen und Beamte am Rande des Demonstrationszuges und von Veranstaltungen generieren gleichfalls Filmund Tonaufnahmen, sobald Erkenntnisse vorliegen, dass auf der Versammlung gewalttätige Ausschreitungen begangen werden. Die Drohne ist somit lediglich ein mögliches Einsatzmittel.

Gleichwohl wäre der bayerische Gesetzgeber gut beraten gewesen, Art. 47 PAG um einen weiteren Passus zu ergänzen. Nämlich, dass sobald ein Drohneneinsatz beabsichtigt ist, der Demonstrations- bzw. Veranstaltungsleiter informiert wird und dieser den Aufstieg der Drohne unverzüglich bekanntzugeben hat. ${ }^{7}$ So hätte man auch dem Argument begegnen können, dass der offene Einsatz der Drohne de facto gar nicht beabsichtigt ist bzw. leicht unterlaufen werden kann. Denn niemand hindert die Polizei daran, die Drohne in einem Hinterhof, auf einem Dach oder in einer unbelebten Straße zwar offen aufsteigen zu lassen, um sodann unentdeckt in $1 \mathrm{~km}$ Entfernung und 700 Meter Höhe Gefährder in einer Demonstration ausfindig zu machen bzw. Ansammlungen aufgrund ihrer Größe zu überwachen. Es besteht folglich die berechtigte Sorge, dass sich der Drohneneinsatz verselbstständigt und weder die Gefährder - was gegebenenfalls zu rechtfertigen wäre - noch die friedlichen Demonstranten überhaupt eine Kenntnis vom Einsatz erlangen.

\section{"Gefährder-Hotspots“ im Visier}

\section{Art. 33 Abs. 2 i. V. m. Art. 13 Abs. 1 Nr. 2, Nr. 3 PAG}

Die bayerische Polizei beabsichtigt zudem gemäß Art. 13 Abs. 1 Nr. 2 (Abb. 3) Drohnen im Zuge der Gefahrenabwehr über bestimmten Örtlichkeiten einzusetzen, um einer Gefahr oder einer drohenden Gefahr für ein bedeutendes Rechtsgut entgegenzu-

7 Entsprechend §1 Abs. 3 Gesetz über Aufnahmen und Aufzeichnungen von Bild und Ton bei Versammlungen unter freiem Himmel und Aufzügen vom 23. April 2013 (Berlin).

\section{Art. 33 Offene Bild- und Tonaufnahmen}

(1) Die Polizei kann bei oder im Zusammenhang mit öffentlichen Veranstaltungen oder Ansammlungen personenbezogene Daten offen

1. auch durch den Einsatz technischer Mittel zur Anfertigung von Bild- und Tonaufnahmen oder -aufzeichnungen über die für eine Gefahr Verantwortlichen erheben, wenn tatsächliche Anhaltspunkte die Annahme rechtfertigen, dass dabei Ordnungswidrigkeiten von erheblicher Bedeutung oder Straftaten begangen werden, oder

2. mittels
a) Bildaufnahmen oder Übersichtsaufnahmen oder
b) Übersichtsaufzeichnungen

erheben, wenn dies wegen der Größe oder Unübersichtlichkeit der Örtlichkeit erforderlich ist; die gezielte Feststellung der Identität einer auf der Übersichtsaufzeichnung abgebildeten Person ist nur unter den Voraussetzungen der Nr.1 zulässig.

(2) Die Polizei kann

1. zur Abwehr

a) einer Gefahr oder

b) einer drohenden Gefahr für ein bedeutendes Rechtsgut,

2. an den in Art. 13 Abs. 1 Nr. 2 genannten Orten, wenn sie öffentlich zugänglich sind, oder

3. an Orten, bei denen tatsächliche Anhaltspunkte die Annahme rechtfertigen, dass dort Ordnungswidrigkeiten von erheblicher Bedeutung oder Straftaten begangen werden, wenn diese Orte öffentlich zugänglich sind,

offen Bild- und Tonaufnahmen oder -aufzeichnungen von Personen anfertigen.

(3) Die Polizei kann an oder in den in Art. 13 Abs. 1 Nr. 3 genannten Objekten offen Bild- und Tonaufnahmen oder -aufzeichnungen von Personen anfertigen, soweit tatsächliche Anhaltspunkte die Annahme rechtfertigen, daß an oder in Objekten dieser Art Straftaten begangen werden sollen, durch die Personen, diese Objekte oder andere darin befindliche Sachen gefährdet sind.

$(\ldots)$

Abb. 2: Art. 33 Abs.1-3 Bayerisches Polizeiaufgabengesetz.

Quelle: http://www.gesetze-bayern.de/Content/Document/BayPAG-47

wirken. Unter einer Gefahr versteht die Rechtsprechung einen Lebenssachverhalt, in dessen ungehindertem Ablauf in absehbarer Zeit mit hinreichender Wahrscheinlichkeit ein Schaden an polizeilichen bzw. ordnungsrechtlichen Gütern eintreten wird (Schmidt 2017, S. 245). Entscheidend ist somit der Zeitfaktor. In der juristischen Kasuistik wird folglich zwischen der ,konkreten Gefahr“ und der ,abstrakten Gefahr“ getrennt. So verlangt die „konkrete Gefahr" eine besondere zeitliche Nähe des vorhandenen Schadensereignisses, dass bereits in Teilaspekten eingetreten ist oder in seiner Realisierung unmittelbar bevorsteht (ebd., S. 245). Hingegen ist eine ,,abstrakte Gefahr“" in der Regel gegeben, wenn ein Sachverhalt bei generell typischer Betrachtungs- 
weise regelmäßig in ein Schadensereignis mündet (ebd., S. 246). Der bayerische Gesetzgeber hat diesem Gefahrenbegriff in $\mathrm{Zu}-$ kunft einen weiteren hinzugefügt: Die „drohende Gefahr“. Mit dieser Begrifflichkeit sollen Vorfeldtatbestände erfasst werden. Dies ist indessen umstritten, denn das Bundesverfassungsgericht hat diese Ausweitung des Gefahrentatbestandes im sogenannten BKA-Urteil zunächst einmal wohl nur im Zusammenhang mit terroristischen Straftaten als rechtmäßig angesehen. ${ }^{8}$

Bayern hat diesen Aspekt der „drohenden Gefahr" in mehrfacher Hinsicht aufgegriffen, erweitert und neu interpretiert (Müller 2018, S. 113). Gemäß der Lesart des bayerischen Gesetzgebers habe das höchste deutsche Gericht ausdrücklich klargestellt, dass die Verfassung den Gesetzgeber nicht von vornherein darin beschränkt, Eingriffstatbestände nur zur Abwehr konkreter, unmittelbar bevorstehender oder gegenwärtiger terroristischer Gefahren zu erlassen. Vielmehr kann er die Grenzen für bestimmte Bereiche mit dem Ziel der Strafverhütung auch weiterziehen, indem er die Anforderungen an die Vorhersehbarkeit des Kausalverlaufes reduziert (ebd., S. 113). Dies ist zwar richtig, jedoch unterstreicht das Bundesverfassungsgericht explizit, dass allgemeine Erfahrungssätze alleine eben nicht ausreichen, um den Zugriff zu rechtfertigen. ${ }^{9}$

Fakt ist jedoch, dass in Bayern ab sofort der offene Einsatz von Drohnen an Örtlichkeiten erlaubt ist, wo sich Personen aufhalten, von denen eine Gefahr bzw. eine drohende Gefahr ausgehen könnte. Als Örtlichkeiten kommen gemäß Art. 13 Abs. 1 Nr. 2 und Nr. 3 PAG eine beträchtliche Anzahl von Hotspots für Straftaten in Betracht: Hauptbahnhöfe und die Umgebung, Ausländertreffpunkte, Rotlichtbezirke, Asylbewerberunterkünfte, Verkehrs- oder Versorgungsanlagen oder -einrichtungen, öffentlichen Verkehrsmittel, Amtsgebäude oder anderen besonders gefährdeten Orten. Dies jedoch immer unter der folgenden Prämisse: Der Drohneneinsatz muss „offen“ erfolgen, es muss eine konkrete Gefahrensituation bestehen oder aber eine Gefahr von diesem Personenkreis drohen. Dass die Offenheit des Drohneneinsatzes - wie bereits dargestellt - einen Widerspruch in sich selbst darstellt, hat der Gesetzgeber ignoriert.

\section{Bewaffnungsverbot für Drohnen}

Gemäß Art. 47 Abs. 4 Bayerisches Polizeiaufgabengesetz ist eine Bewaffnung von Polizeidrohnen verboten. Folglich verbietet sich eine Bestückung der Drohne mit Pfefferspray, Tränengas, Tasern oder sogar Schusswaffen. Diese Klarstellung, die im ersten Gesetzesentwurf noch fehlte, war in der Tat geboten, denn das PAG verwendet einen offenen Technikbegriff.

Während zum Beispiel in Art. 78 PAG eine abschließende Aufzählung bezüglich der im Einsatz zu verwendenden Waffen durch die Polizei erfolgt, findet sich in Art. 47 PAG keine Festlegung der Drohnensysteme bzw. Drohnentypen. Die momen-

8 BVerfGE, in NJW 2016, S. $1781 \mathrm{ff}$ (sog. BKA-Urteil).

9 BVerfGE, in NJW 2016, S. $1781 \mathrm{ff}$ (S. 1785) (sog. BKA-Urteil).
Art. 13 Identitätsfeststellung und Prüfung von Berechtigungsscheinen

(1) Die Polizei kann die Identität einer Person feststellen

1. zur Abwehr

a) einer Gefahr oder

b) einer drohenden Gefahr für ein bedeutendes Rechtsgut,

2. wenn die Person sich an einem Ort aufhält,

a) von dem auf Grund tatsächlicher Anhaltspunkte anzunehmen ist, daß dort

aa) Personen Straftaten verabreden, vorbereiten oder verüben,

bb) sich Personen ohne erforderliche Aufenthaltserlaubnis treffen, oder

cc) sich Straftäter verbergen, oder

b) an dem Personen der Prostitution nachgehen, oder

c) der als Unterkunft oder dem sonstigen, auch vorübergehenden Aufenthalt von Asylbewerbern und unerlaubt Aufhältigen dient,

3. wenn sie sich in einer Verkehrs- oder Versorgungsanlage oder -einrichtung, einem öffentlichen Verkehrsmittel, Amtsgebäude oder einem anderen besonders gefährdeten Objekt oder in unmittelbarer Nähe hiervon aufhält und Tatsachen die Annahme rechtfertigen, daß in oder an Objekten dieser Art Straftaten begangen werden sollen, durch die in oder an diesen Objekten befindliche Personen oder diese Objekte selbst unmittelbar gefährdet sind,

$(\ldots)$

Abb.3: Art.13 Abs.1 Nr.1-3 Bayerisches Polizeiaufgabengesetz.

Quelle: http://www.gesetze-bayern.de/Content/Document/BayPAG-47

tane Verwendung von Quadro-, Hexa- und Oktakoptern durch die Länderpolizeien - die nur eine Nutzlast von maximal ca. $3 \mathrm{~kg}$ tragen können - würde zwar ohnehin nur eine leichte Bewaffnung wie etwa mit Pfefferspray oder Tränengas erlauben. Dass diese Ausrüstung jedoch äußerst zielgerichtet eingesetzt werden kann demonstriert die israelische Grenzpolizei am Zaun des Gaza-Streifens. Dort kommen bereits relativ kleine Drohnensysteme zum Einsatz und versprühen Tränengas (Times of Israel 2018).

Völlig andere Möglichkeiten würden sich aber ergeben, wenn die Polizei in Zukunft großen Systemen den Vorzug geben würde. Drohnen der Klasse „Predator“ oder „Pegasus“ verfügen über eine Traglast von $2.000 \mathrm{~kg}$ und über die Kapazität, in 5,5 km Flughöhe mithilfe von 368 Chips Aufnahmen zu fertigen, die mit einer Bildqualität von 1,8 Billionen Pixel ein über 40 Quadratkilometer großes Untersuchungsgebiet abdecken und dabei jeden Tag rund 1 Million Terrabyte an Daten produzieren (Tuck 2016, S. 76; Jaeger 2017, S. 15). Angesichts dieser technischen Dimensionen muten die fragilen deutschen Polizeidrohnen wie Spielzeug an. Hier ist jedoch Vorsicht geboten, denn gerade die Entwicklung sogenannter Mikrowaffen schreitet rapide voran. Diese Drohnen, die kleiner sind als Hummeln und durchaus Sprengladungen tragen und im Schwarm auftreten können, sind zwar noch als Schwellentechnologie zu qualifizieren, sie vermitteln aber einen Ausblick auf technische Zukunftsszenarien (Tuck 2016, S. 86). 


\section{Fazit: Vorsätzliche Grenzüberschreitung}

Der Einsatz von Polizeidrohnen ist differenziert zu betrachten. Tatortsicherung, Verkehrsraumüberwachung und die Suche nach Vermissten bereiten keine verfassungsrechtlichen und damit rechtspolitischen Probleme. Hingegen sind gezielte Überwachungsmaßnahmen von Orten, Personen bzw. Personenansammlungen völlig anders zu bewerten. Der bayerische Gesetzgeber macht es sich zu einfach, wenn er den Chilling Effect durch Kleidervorschriften für „Drohnenpolizisten“ oder ein Hinweisschild am Eingang einer öffentlichen Veranstaltung beseitigen will. Im Spannungsfeld von öffentlicher Sicherheit und persönlicher Freiheit bedarf es ausgefeilterer Regelungen. Hinzu kommt, dass Drohnen weder mit den lauten Polizeihubschraubern noch mit einem polizeilichen Überwachungswagen zu vergleichen sind. Es handelt sich um eine völlig neue Technologie mit hohem technischem Potenzial. Bereits die Festlegung, dass Drohnen ,offen“ aufzusteigen haben, verdeutlicht die Problematik. Sobald die Drohne in die Höhe schwebt, kann keine Offenheit mehr garantiert werden - und soll es im Übrigen wohl auch nicht. In jedem Fall hat der bayerische Gesetzgeber ganz bewusst Grenzen überschritten, die in den anstehenden Klageverfahren durch die Richter des Bayerischen Verfassungsgerichtsund des Bundesverfassungsgerichts bewertet werden müssen.

\section{Literatur}

Abgeordnetenhaus Berlin (2016): Wortprotokoll. Ausschuss für öffentliche Sicherheit und Ordnung. 80. Sitzung, 20.06.2016. Online verfügbar unter https://www.parlament-berlin.de/ados/17/InnSich0/protokoll/iso17080-wp.pdf, zuletzt geprüft am 18. 07.2018.

Assion, Simon (2014): Was sagt die Rechtsprechung zu Chilling Effects? In: Telemedicus. Recht der Informationsgesellschaft, 09. 05.2014. Online verfügbar unter https://www.telemedicus.info/article/2773-Was-sagtdie-Rechtsprechung-zu-Chilling-Effects.html, zuletzt geprüft am 18. 07.2018.

Bayerischer Landtag (2018 a): Wortprotokoll. Ausschuss für kommunale Fragen, Innere Sicherheit und Sport, 90. Sitzung sowie Ausschuss für Verfassung, Recht und Parlamentsfragen, 86. Sitzung, 21.03.2018, S. 23-24. Online verfügbar unter https://asjbayern.de/workspace/media/ static/090ki210318gesendgkopie-5adf3aee03929.pdf, zuletzt geprüft am 15.10.2018.

Bayerischer Landtag (2018 b): Wortprotokoll. Ausschuss für kommunale Fragen, Innere Sicherheit und Sport. 90. Sitzung sowie Ausschuss für Verfassung, Recht und Parlamentsfragen, 86. Sitzung, 21. 03.2018.

Bayerischer Landtag (2018c): Gesetzentwurf der Staatsregierung für ein Gesetz zur Neuordnung des bayerischen Polizeirechts (PAG-Neuordnungsgesetz). Drucksache 17/20425. Online verfügbar unter https://www.bayern. landtag.de/www/ElanTextAblage_WP17/Drucksachen/Basisdrucksac hen/0000013000/0000013038.pdf, zuletzt geprüft am 15.10.2018.

Bremische Bürgerschaft (2014): Plenarprotokoll, Landtag, 18. WP, 62. Sitzung, 19. 06.2014. Online verfügbar unter http://www.bremische-buergerschaft. de/dokumente/wp18/land/protokoll/P18L0062.pdf, zuletzt geprüft am 15. 10.2018.

Diehl, Jörg (2010): Polizei-Drohnen. Himmelfahrtskommando für die Schönwetterspäher. In: Spiegel Online, 22.06.2010. Online verfügbar unter http://www.spiegel.de/panorama/polizei-drohnen-himmelfahrtskommandofuer-die-schoenwetterspaeher-a-701310.html, zuletzt geprüft am 18. 07.2018. Gusy, Christoph (2014): Aufklärungsdrohnen im Polizeieinsatz. In: Die Kriminalpolizei, Ausgabe März/2014. Online verfügbar unter https://www. kriminalpolizei.de/ausgaben/2014/maerz/detailansicht-maerz/artikel/ aufklaerungsdrohnen-im-polizeieinsatz.html, zuletzt geprüft am 18.07.2018. Hertwig, Jana; Damla, Kuvvet (2011): Technik vs. Recht. Verfassungs- und polizeirechtliche Probleme des Einsatzes von Aufklärungsdrohnen in Deutschland am Beispiel des Castor-Transportes nach Gorleben im November 2010. In: Humanitäres Völkerrecht Informationsschriften 24 (02), S. 120-127.

Jaeger, Rolf Rainer (2017): Künstliche Intelligenz. Hilfsmittel oder Konkurrenz für die Polizei. In: Der Kriminalist 2017 (3), S. 8-15.

Kornmeier, Claudia (2018): Übrigens nicht bewaffnet. Bayerisches PAG zu Drohnen-Einsatz. In: Legal Tribune Online, 30.05. 2018. Online verfügbar unter https://www.lto.de/recht/hintergruende/h/bayern-pag-reform-drohneneinsatz-ueberwachung-grundrechte/, zuletzt geprüft am 18.07.2018.

Müller, Birgit (2018): Das Gesetz zur effektiveren Überwachung gefährlicher Personen und die daraus erwachsenen neuen Befugnisse der Bayerischen Polizei. In: Bayerische Verwaltungsblätter, Heft 4/2018, S. 109-116.

Schmidt, Rolf (2017): Polizei- und Ordnungsrecht. Grasberg: Fachverlag Rolf Schmidt GmbH.

Schulz, André (2017): Künstliche Intelligenz. Hilfsmittel oder Konkurrenz für die Polizei. In: Der Kriminalist 2017 (3), S. 16-18.

Times of Israel (2018): Israel deploys drones to drop tear gas on Gaza protesters. Friday's violence marked one of the first uses of the new technology, Border Police spokesman says, 31. 03.2018. Online verfügbar unter https://www.timesofisrael.com/israel-deploys-drones-to-drop-tear-gason-gaza-protesters, zuletzt geprüft am 18.07.2018.

Truscheit, Karin (2017): Leiser als Hubschrauber. Drohnen bei der Polizei. In: Frankfurter Allgemeine Zeitung, 20.11.2017. Online verfügbar unter http:// www.faz.net/aktuell/gesellschaft/kriminalitaet/die-bayerische-polizei-willkuenftig-mehr-drohnen-nutzen-15301940.html, zuletzt geprüft am 18. 07.2018. Tuck, Jay (2016): Evolution ohne uns. Kulmbach: Plassen Verlag. Weiner, Bernhard (2018): Kommentierung zu §31 Niedersächsisches Sicherheitsund Ordnungsgesetz. In: Markus Möstl und Bernhard Weiner (Hg.): Beck'scher Online-Kommentar Polizei- und Ordnungsrecht Niedersachsen, 11. Edition, Stand: 1. 8.2018, München 2018.

Zöller, Mark; Saleh, Ihwas (2014): Rechtliche Rahmenbedingungen des polizeilichen Flugdrohneneinsatzes. In: Neue Zeitschrift für Verwaltungsrecht 33 (7), S. 408-414.

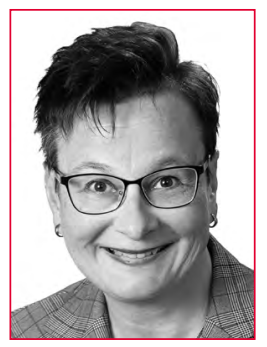

PROF.DR.JUR. SUSANNE BENÖHRLAQUEUR

arbeitet nach Tätigkeiten als Rechtsanwältin, Universitäts- und Fachhochschuldozentin, Vertretungsprofessorin sowie Umweltdezernentin und Stadträtin seit 2018 als Professorin für Staatsund Eingriffsrecht an der Fachhochschule für öffentliche Verwaltung NRW am Standort Münster. 